\begin{tabular}{|c|l|}
\hline Title & Polarization-dependent photoinduced mechanical deformations in coval ent chal cogenide glasses \\
\hline Author(s) & A sao, Hideaki; Tanaka, Keiji \\
\hline Citation & $\begin{array}{l}\text { Journal of A pplied Physics, 102(4), 043508 } \\
\text { https://doi.org/10.1063/1.2770816 }\end{array}$ \\
\hline Issue Date & 2007-08-15 \\
\hline Doc URL & http://hdl.handle.net/2115/30159 \\
\hline Rights & Copyright $\odot 2007$ American Institute of Physics \\
\hline Type & article \\
\hline File Information & JAP102-04.pdf \\
\hline
\end{tabular}

Instructions for use 


\title{
Polarization-dependent photoinduced mechanical deformations in covalent chalcogenide glasses
}

\author{
Hideaki Asao and Keiji Tanaka ${ }^{a)}$ \\ Department of Applied Physics, Graduate School of Engineering, Hokkaido University, \\ Sapporo 060-8628, Japan
}

(Received 4 May 2007; accepted 3 July 2007; published online 21 August 2007)

\begin{abstract}
The optomechanical effect and photoinduced anisotropic deformation induced by illumination of linearly polarized light have been comparatively studied for glassy $\mathrm{As}_{2} \mathrm{~S}_{3}$ and Se films. The two materials show qualitatively the same behaviors in the mechanical effect and the deformation, which suggests that these photoinduced phenomena are inherent to the covalent chalcogenide glass. But, the two phenomena show different dependences upon intensity, spectrum and exposure time of excitation light, which imply different underlying mechanisms. (c) 2007 American Institute of Physics. [DOI: 10.1063/1.2770816]
\end{abstract}

\section{INTRODUCTION}

Covalent chalcogenide glasses have been known to exhibit a variety of photoinduced phenomena. ${ }^{1-3}$ For instance, illumination at room temperature to annealed $\mathrm{As}_{2} \mathrm{~S}_{3}$ films provides photodarkening (band gap reduction) by $\sim 2 \%,{ }^{4}$ volume expansion by $\sim 0.4 \%,{ }^{5}$ refractive-index increase by $\sim 1 \%,{ }^{6}$ elastic-constant reduction by $\sim 8 \%,{ }^{7}$ and so forth. ${ }^{1-3}$ Since these modifications are induced efficiently by illumination of the so-called band gap light with photon energy of $\hbar \omega \approx E_{g}$, where $E_{g}$ is an optical gap of the material of interest, ${ }^{8}$ it is undoubtful that photoelectronic excitations trigger successive electrostructural changes. However, it is difficult to identify the structural change, since the glass structure is disordered. ${ }^{2,3}$ In addition, in some phenomena, illumination of linearly polarized light provides anisotropic changes, which are referred to as vectoral. ${ }^{1,2,9,10}$ Not only the photon energy but also the direction of electric fields exerts some influence on the photostructural processes. Known examples are photoinduced dichroism, ${ }^{11}$ birefringence, ${ }^{11}$ and anisotropic crystallization. ${ }^{12}$ Note that the polarization dependence implicitly suggests that temperature rises necessarily occurring at light absorption cannot be responsible for such phenomena. It should be mentioned that oxide glasses and organic polymers exhibit similar phenomena, ${ }^{1,3,13,14}$ and accordingly, a deeper understanding of photostructural processes in noncrystalline materials is an important subject in solid state science.

Recently, Krecmer et al. ${ }^{15}$ and Saliminia et al. ${ }^{16}$ have discovered two vectoral phenomena which produce macroscopic deformations. One is an optomechanical (OM) effect, ${ }^{15,17-20}$ which is a polarization-dependent deflection of small bilayer cantilevers consisting of As-S-Se and $\mathrm{Si}$ (or $\mathrm{Si}_{3} \mathrm{~N}_{4}$ ). A similar phenomenon is reported also for $\mathrm{As}_{50} \mathrm{Se}_{50} /$ mica cantilevers by Trunov and Bilanich. ${ }^{21}$ The other is a photoinduced anisotropic M-shaped deformation in $\mathrm{As}_{2} \mathrm{~S}_{3}$ films. ${ }^{16,22,23}$ Both of these phenomena are induced by illumination of linearly polarized band gap light. On the other hand, the OM effect appears only during illumination,

${ }^{a)}$ Electronic mail: keiji@eng.hokudai.ac.jp i.e., it is transitory, while the anisotropic deformation exists in quasistable after illumination. In addition, the deflection and the deformation have been induced, respectively, by unfocused and focused laser beams.

Are these vectoral phenomena related? Since both the phenomena are vectoral and mechanical, we may envisage some interrelation. The transitory OM motion may cause the anisotropic deformation. However, such a problem remains to be studied. We are also interested in a problem if these phenomena appear in amorphous $\mathrm{Se}$, which has a low glasstransition temperature of $\sim 40{ }^{\circ} \mathrm{C}$ and an atomic structure consisting of entangled chains, which are substantially different from the glass-transition temperature of $\sim 200{ }^{\circ} \mathrm{C}$ and cross-linked structures in $\mathrm{As}_{2} \mathrm{~S}(\mathrm{Se})_{3}{ }^{2}{ }^{2}$ Actually, photodarkening in Se can be detected only at low temperatures. ${ }^{24}$ In the present study, therefore, following a previous work on microscopic mechanisms of the photoinduced deformation in $\mathrm{As}_{2} \mathrm{~S}_{3}$, ${ }^{25}$ we will investigate these problems for these elemental and stoichiometric materials. The OM effect will be measured in somewhat different ways from those employed previously. ${ }^{15,17-20}$

\section{EXPERIMENTS}

Four kinds of samples were prepared by thermal evaporation of glassy $\mathrm{As}_{2} \mathrm{~S}_{3}$ and $\mathrm{Se}$ onto oxide-glass and cleavedmica $\left(\sim 5-\mu \mathrm{m}\right.$ thick) substrates. Deposited $\mathrm{As}_{2} \mathrm{~S}_{3}$ and $\mathrm{Se}$ films with thicknesses ranging from 0.3 to $7 \mu \mathrm{m}$ were, respectively, annealed at $\sim 180{ }^{\circ} \mathrm{C}$ and stored at room temperature for thermal stabilization. Mica samples were then cut into rectangular forms with a typical dimension of 0.1 $\times 1 \mathrm{~mm}^{2}$ for investigation of the OM effect.

These samples were excited by linearly polarized laser beams with photon energies of $1.8,2.0$, and $2.3 \mathrm{eV}$. Note these energies are located at around Tauc optical gaps of 2.4 $\mathrm{eV}$ in $\mathrm{As}_{2} \mathrm{~S}_{3}$ and $2.0 \mathrm{eV}$ in $\mathrm{Se}^{2,8}$ Photoinduced anisotropic deformations were investigated following the same procedure as that employed previously. ${ }^{25}$ In short, the chalcogenide film was irradiated by the laser beam, which was focused to 5-10 $\mu \mathrm{m}$ in spot diameter, and then, irradiated areas 

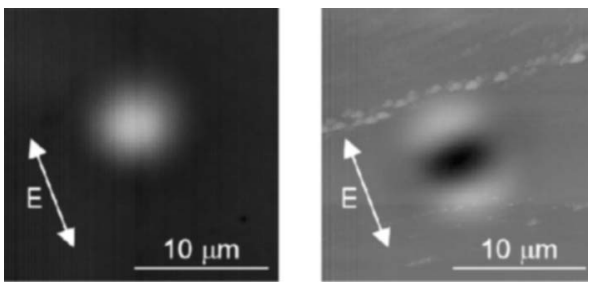

FIG. 1. AFM images of (a) an isotropic expansion and (b) an anisotropic deformation in Se films with thicknesses of (a) $\sim 7$ and (b) $\sim 0.7 \mu \mathrm{m}$ after exposures of 60 and $300 \mathrm{~s}$, respectively. The illumination source is a linearly polarized He-Ne laser $(2.0 \mathrm{eV})$, which gives an irradiance of $\sim 400 \mathrm{~W} / \mathrm{cm}^{2}$ in a focused spot of $\sim 5 \mu \mathrm{m}$ in diameter. The double arrows show the polarization direction.

were imaged using an atomic force microscope (AFM). In addition, growing dynamics of deformations were inspected, in situ, through transmitted light patterns, which were monitored using a charge coupled device (Ophir, BeamStar FX50), the method previously being employed for an investigation of optical damages. ${ }^{26}$ For measurements of the OM effect, the mica sample was fixed to a glass slide using an viscous grease as a cantilever, which was exposed to an unfocused laser beam, unless otherwise noted. The polarization direction was varied using a quarter-wavelength plate and a polarizer. Changes in light intensities upon polarization rotation were smaller than 5\%, which could be neglected. Resulting cantilever deflections were evaluated through direct measurements of displacements of a cantilever apex using an optical microscope having a microscale. Or, when the apex displacement was smaller than $\sim 2 \mu \mathrm{m}$, which was the case for Se, an optical lever technique was employed, in which an excitation laser beam worked also as a probe. The beam, after being reflected from the cantilever, was detected by the imaging device, and the spot positions were converted to cantilever deflections. All photoexcitations were provided at room temperature, except for some optical-lever experiments of Se samples, which were held in an optical cryostat at 80 $\mathrm{K}$.

\section{RESULTS}

\section{A. Anisotropic deformation}

Isotropic and anisotropic deformations in $\mathrm{As}_{2} \mathrm{~S}_{3}$ have appeared irrespective of the substrates. In the previous studies, ${ }^{16,22,23,25} \mathrm{As}_{2} \mathrm{~S}_{3}$ films were deposited onto microscope slides, which were exposed to focused linearly polarized laser beams. It was demonstrated that an isotropic expansion appears at first, which gradually transforms to an anisotropic M-shaped deformation with exposure time. The present study has confirmed these features for $\mathrm{As}_{2} \mathrm{~S}_{3}$ films deposited on mica substrates.

A remaining problem is if such deformations appear in Se. Since the glass-transition temperature of $\mathrm{Se}$ is $\sim 40{ }^{\circ} \mathrm{C},{ }^{2}$ just above room temperature, we may assume that the deformation disappears within short intervals after terminating exposures. By contrast, Fig. 1 manifests that (a) isotropic expansions and (b) anisotropic M-shaped deformations appear in thick $(\sim 7 \mu \mathrm{m})$ and thin $(\sim 0.7 \mu \mathrm{m})$ Se films after illumination of focused linearly polarized light of $2.0 \mathrm{eV}$ for 1 and 5 min. It should be mentioned that subgap illumination with a photon energy of $1.8 \mathrm{eV}$ has also produced similar isotropic and anisotropic deformations in Se films.

The expansion in (a) has a central height of $\sim 90 \mathrm{~nm}$ and a lateral extension of $\sim 5 \mu \mathrm{m}$, which corresponds to a spot diameter $2 r$ of the excitation light. Since $2 r$ is comparable to the film thickness $L(\approx 7 \mu \mathrm{m})$, the expansion can be regarded as giant, ${ }^{1-3,27,28}$ in which the apparent fractional expansion $\Delta L / L(\approx 90 \mathrm{~nm} / 7 \mu \mathrm{m} \approx 1 \%)$ can be written as $\Delta L / L=(1+L / r)\left(\Delta L_{0} / L\right)$, where $\Delta L_{0} / L$ is an intrinsic fractional expansion. This equation gives $\Delta L_{0} / L \approx 0.3 \%$ for Se, which is, surprisingly, similar to that $(\sim 0.4 \%)$ in $\mathrm{As}_{2} \mathrm{~S}_{3}{ }^{3,5,27,28}$ The expansion shape is unchanged upon prolonged exposures up to $10 \mathrm{~min}$, being consistent with the previous observation for thick $\mathrm{As}_{2} \mathrm{~S}_{3}$ films of $2 r \lesssim \Delta L{ }^{25}$ This isotropic expansion may have a common origin to that of relief fringes produced holographically in Se films. ${ }^{29}$

On the other hand, when $2 r \geq L$ as in (b), the anisotropic M-shaped deformation becomes prominent. ${ }^{25}$ The central valley and the peripheral peaks are lower and higher by 120 $\mathrm{nm}$ and $\sim 50 \mathrm{~nm}$ than the original film surface, i.e., a peakto-valley difference $h$ being $\sim 170 \mathrm{~nm}$. In this case, following the previous study, ${ }^{25}$ we expect that an isotropic expansion occurs in advance to this anisotropic deformation. However, the estimated $\Delta L_{0} / L$ of $\sim 0.3 \%$ suggests that the isotropic expansion has a height of $\sim 2 \mathrm{~nm}$ for this thin film, which could not be detected due to image noises and, probably, due to faster relaxation of the isotropic deformation than that of the anisotropic. ${ }^{25}$

In short, the two kinds of deformations appear in Se in similar ways to those in $\mathrm{As}_{2} \mathrm{~S}_{3}$. Different from undetected photodarkening in Se at room temperature, ${ }^{24}$ these macroscopic deformations seem to exist, at least, one day. These contrastive optical and deformation behaviors are consistent with previous ideas that the photodarkening is atomic ${ }^{1-3}$ while the deformations appear through macroscopic fluidity. ${ }^{25,28}$

Figure 2 shows light-intensity dependence of the peakto-valley height $h$ in anisotropic deformations in $\mathrm{As}_{2} \mathrm{~S}_{3}$ and Se. Naturally, $h$ tends to increase with the absorbed dose $\alpha I t,{ }^{16,25}$ which is proportional to the absorbed photon number, where $\alpha$ is the absorption coefficient, $I$ is the incident light irradiance, and $t$ is the exposure time. Accordingly, in the figure, the light-intensity dependence is compared under fixed absorbed doses. In addition, for comparing the characteristics in $\mathrm{As}_{2} \mathrm{~S}_{3}$ and $\mathrm{Se}$, which are exposed to differentenergy excitations, the horizontal axis is taken to be the absorbed light intensity $\alpha I$.

We see some interesting features. For an $\mathrm{As}_{2} \mathrm{~S}_{3}$ film with a thickness of $2 \mu \mathrm{m}$, subgap $(2.0 \mathrm{eV})$ and band gap $(2.3 \mathrm{eV})$ illumination give nearly straight lines of $h \propto \alpha I$, which resembles that of the photoinduced fluidity $\eta^{-1}$ (solid line), which has been calculated from strain-stress curves of $\mathrm{As}_{2} \mathrm{~S}_{3}$ films exposed to $2.0 \mathrm{eV}$ light. ${ }^{30-32}$ Note that, in this presentation, a reciprocity law between the light intensity and the exposure time demands $h \propto(\alpha I)^{0}$, which is clearly denied. On the other hand, Se seems to show a weaker light-intensity dependence. Nevertheless, interestingly, the absolute magni- 


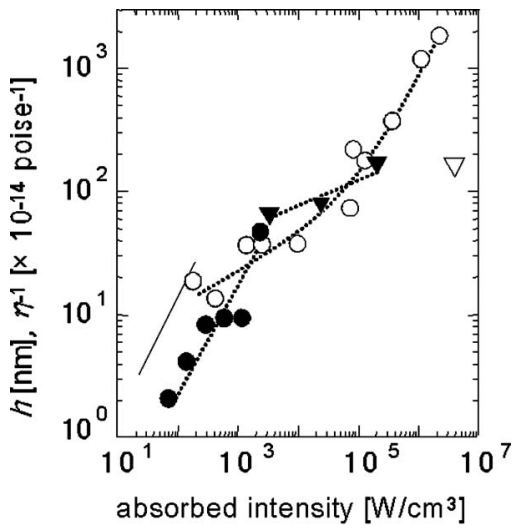

FIG. 2. Absorbed light-intensity $(\alpha I)$ dependence of the peak-to-valley

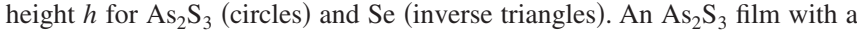
thickness of $2 \mu \mathrm{m}$ is exposed to $2.0 \mathrm{eV}$ light at a dose $\alpha I t$ of 5 $\times 10^{6} \mathrm{~J} / \mathrm{cm}^{3}(\bullet)$ and to $2.3 \mathrm{eV}$ at $2 \times 10^{7} \mathrm{~J} / \mathrm{cm}^{3}(\circ)$. $\nabla$ shows a result for a Se film $\left(0.7-\mu \mathrm{m}\right.$ thick) exposed to $2.0 \mathrm{eV}$ light at $5 \times 10^{7} \mathrm{~J} / \mathrm{cm}^{3}$ [Fig. 1(b)], and three $\boldsymbol{\nabla}$ show for Se $(1-\mu \mathrm{m}$ thick) exposed to $1.8 \mathrm{eV}$ light at 1 $\times 10^{9} \mathrm{~J} / \mathrm{cm}^{3}$. For comparison, light-intensity dependence of photoinduced fluidity $\eta^{-1}$ in $\mathrm{As}_{2} \mathrm{~S}_{3}$ induced by $2.0 \mathrm{eV}$ light is shown by a solid line (Ref. $31)$.

tude of $h$ for $1.8 \mathrm{eV}$ exposures is comparable to that in $\mathrm{As}_{2} \mathrm{~S}_{3}$. Thermal relaxation in Se seems to be neglected for the anisotropic deformation.

\section{B. Optomechanical effect}

Figure 3 shows time variations of the apex displacement $\Delta$ of (a) $\mathrm{As}_{2} \mathrm{~S}_{3}$ - and (b) Se-mica cantilevers upon exposures to linearly polarized band gap light. The zero displacement refers to the initial state before illumination.

Observations in (a) $\mathrm{As}_{2} \mathrm{~S}_{3}$ are qualitatively consistent with those reported previously. ${ }^{15,17-21}$ When linearly polarized illumination is started at $0 \mathrm{~min}$, irrespective of the polarization direction, the cantilever bends as if the chalcogenide film expands $(\Delta \approx 110 \mu \mathrm{m})$, which possibly reflects the isotropic expansion [Fig. 1(a)], a difference of thermal expansions between $\mathrm{As}_{2} \mathrm{~S}_{3}$ and substrates, ${ }^{20}$ and photoinduced stress relaxation. ${ }^{33}$ After this initial response, the electric field of linearly polarized light perpendicular $(\perp)$ and parallel $(\|)$ to the cantilever long-axis enhances and suppresses the displacement with modulation of $\Delta \approx 10 \mu \mathrm{m}$. When the light is turned off, the displacement slowly relaxes. We also see in this trace that the OM displacement of $\sim 10 \mu \mathrm{m}$ is nearly

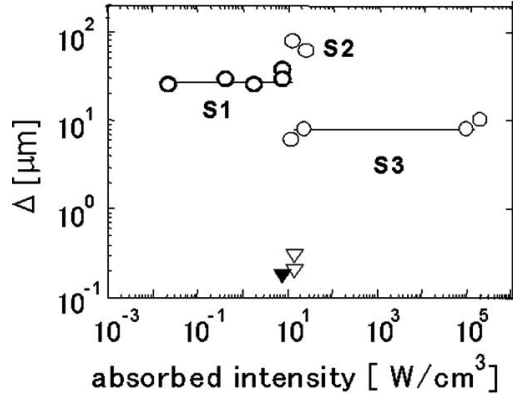

FIG. 4. Optomechanical displacements $\Delta$ at room temperature as a function of absorbed light intensity $\alpha I$ in three $\mathrm{As}_{2} \mathrm{~S}_{3}$ cantilevers exposed to $2.3 \mathrm{eV}$ (circles) and a Se cantilever to $2.0 \mathrm{eV}$ (open triangles) and $1.8 \mathrm{eV}$ (closed triangle). S1-S3 denote the $\mathrm{As}_{2} \mathrm{~S}_{3}$ samples listed in Table I. The Se sample has a lateral dimension of $800 \times 60 \mu \mathrm{m}^{2}$ and a film thickness of $5 \mu \mathrm{m}$.

independent of the incident light intensity, the detail being shown in Fig. 4, which is consistent with the previous result. $^{17-20}$

In the case of (b) Se, relaxation under illumination, known as photoinduced stress-relaxation, ${ }^{33}$ is remarkable. But, we see that perpendicular and parallel polarization tend to lengthen and shorten Se films along the cantilever axis, in a similar way to that in (a) $\mathrm{As}_{2} \mathrm{~S}_{3}$. Quantitatively, however, as shown from the different vertical scales in (a) and (b), the Se cantilever provides much smaller displacements. Upon exposures at $80 \mathrm{~K}$ (not shown), the stress relaxation is largely suppressed, and the displacement is enhanced by $\sim 3$ times for $2.0 \mathrm{eV}$ excitation (see, Fig. 6). In short, Se exhibits the OM effect, which is smaller than that in $\mathrm{As}_{2} \mathrm{~S}_{3}$.

A comparison of (a) and (b) gives an important insight. It is clear from (a) that the perpendicular polarization produces greater cantilever deflection, which probably arises from elongation of the chalcogenide films along the cantilever axis. However, in (a) and in all the previous studies, ${ }^{15,17-21}$ it has not been known if the parallel polarization produces real film contractions along the axis, since the polarization-dependent displacement is biased by the greater initial response, i.e., $110 \gg 10 \mu \mathrm{m}$ in Fig. 3(a). In contrast, the result in (b) Se manifests that the parallel polarization provides a purely negative displacement of $-0.3 \mu \mathrm{m}$ at 140 min, which strongly suggests the existence of contracting forces under illumination.

In accordance with the previous result, ${ }^{20}$ the OM deflection is dependent upon cantilever dimensions and a chalcogenide thickness. Table I shows for $\mathrm{As}_{2} \mathrm{~S}_{3}$ cantilevers with a
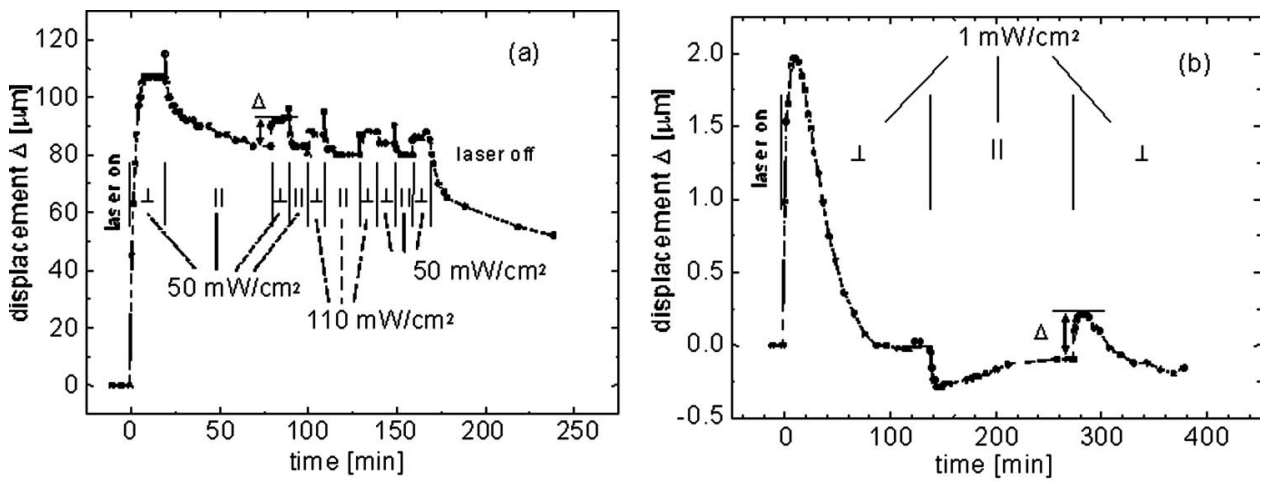

FIG. 3. Cantilever-apex displacements $\Delta$ in (a) $\mathrm{As}_{2} \mathrm{~S}_{3}(0.3 \mu \mathrm{m}) / \mathrm{mica}(5 \mu \mathrm{m})$ with a lateral size of $1500 \times 80 \mu \mathrm{m}^{2}$ (S3 in Table I) upon exposure to 2.3 $\mathrm{eV}$ light and (b) $\operatorname{Se}(5 \mu \mathrm{m}) / \operatorname{mica}(5 \mu \mathrm{m})$ with a lateral size of $800 \times 60 \mu \mathrm{m}^{2}$ to $2.0 \mathrm{eV}$ light. The symbols $\perp$ and $\|$ depict the polarization direction of excitation light perpendicular and parallel to a cantilever long axis. 
TABLE I. Dimensions of three $\mathrm{As}_{2} \mathrm{~S}_{3}$ cantilevers and optomechanical displacement $\Delta . Z$ is the cantilever length, $W$ is the width, and $L$ is the $\mathrm{As}_{2} \mathrm{~S}_{3}$ thickness. The mica substrate is $\sim 5 \mu \mathrm{m}$ in thickness.

\begin{tabular}{ccccccc}
\hline \hline Sample & $Z(\mu \mathrm{m})$ & $W(\mu \mathrm{m})$ & $L(\mu \mathrm{m})$ & $Z L / W(\mu \mathrm{m})$ & $\Delta(\mu \mathrm{m})$ & $\Delta / Z(\%)$ \\
\hline S1 & 2000 & 300 & 5 & 25 & 35 & 1.7 \\
S2 & 1900 & 120 & 5 & 80 & 80 & 4.2 \\
S3 & 1500 & 80 & 0.3 & 8 & 6 & 0.4 \\
\hline \hline
\end{tabular}

length of $Z$ a rough measure of $\Delta / Z \approx 0.4 \%-4 \%$, which is comparable to the maximal $\mathrm{OM}$ displacement of $\sim 2 \%$ in Ref. 20. These values suggest, after a simple geometrical analysis, a corresponding length modulation of the chalcogenide film of $\sim(\Delta / Z)^{2} \approx 10^{-2} \%$, which is smaller by an order than $\Delta L_{0} / L \approx 0.4 \%$ in the intrinsic volume expansion. ${ }^{3,5,27,28}$ In detail, in the present experimental conditions we find an approximate equality, $\Delta \approx Z L / W$, where $L$ is the film thickness and $W$ is the cantilever width. Nevertheless, this equality must be coincidental, since $\Delta$ depends upon other parameters such as the photon energy of excitation (Fig. 6).

Figure 4 shows relations between the OM displacement $\Delta$ and the absorbed light intensity $\alpha I$. As pointed out in Fig. $3, \Delta$ in $\mathrm{As}_{2} \mathrm{~S}_{3}$ (circles) is greater than that (triangles) in Se. We also see for $\mathrm{As}_{2} \mathrm{~S}_{3}$ that $\Delta$ does not change with the light intensity. Here, the data at $\alpha I \leqslant 10^{2} \mathrm{~W} / \mathrm{cm}^{3}$ have been obtained using unfocused $2.3 \mathrm{eV}$ light, which irradiates the cantilevers with nearly spatially uniform intensity distributions, which is also the case in the previous studies. ${ }^{15,17-20}$ On the other hand, the two points for the smallest $\mathrm{As}_{2} \mathrm{~S}_{3}$ cantilever (S3) at $\sim 10^{5} \mathrm{~W} / \mathrm{cm}^{3}$ are obtained using $2.3 \mathrm{eV}$ light, which is focused to a spot of $\sim 30 \mu \mathrm{m}$ in diameter, the size being appreciably smaller than the cantilever width of $80 \mu \mathrm{m}$. However, even for such limited-area irradiations, the independence of $\Delta$ from the light intensity still holds.

Stuchlik et al. have demonstrated that OM transients $\Delta(t)$ follow stretched-exponential type variations. ${ }^{18,20}$ That is, $\Delta(t) \propto 1-\exp \left\{-(t / \tau)^{\beta}\right\}$, where $t$ is an exposure time, $\tau$ is a characteristic time constant, and $\beta$ is the so-called stretching coefficient $(0<\beta \leq 1)$. They report that the transient obtained when changing the polarization from perpendicular to parallel (displacement reduction) can be fitted with two stretched exponentials with different $\tau$ and $\beta$. We then are interested if a similar feature is obtained for the mica cantilevers. In the present experiment, however, since $\Delta$ in $\mathrm{As}_{2} \mathrm{~S}_{3}$ is measured one-by-one using a microscope, only the slow transient at $t>1 \mathrm{~min}$ can be monitored, which gives $\beta_{2}$ $\approx 0.6$ for $\mathrm{S} 3$ at $I=10-100 \mathrm{~mW} / \mathrm{cm}^{2}$. This $\beta_{2}$ is comparable to the previous value of $0.53-0.65,{ }^{20}$ despite of substantial
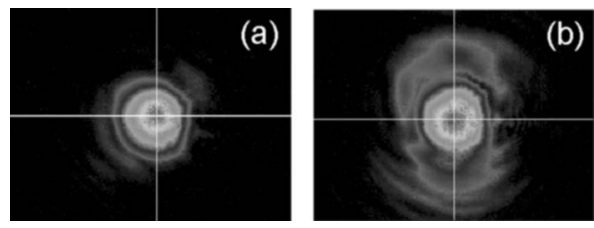

FIG. 5. Transmitted light patterns (a) when illumination is just started and (b) at an exposure time of $20 \mathrm{~min}$. The sample is S3 in Table I, which is exposed to $2.3 \mathrm{eV}$ light with a light spot of $\sim 30 \mu \mathrm{m}$ in diameter and $400 \mathrm{~W} / \mathrm{cm}^{2}$. differences in experimental conditions between the present and the previous study. On the other hand, $\tau$ becomes shorter with an increase in the light intensity (Fig. 7).

\section{Comparison}

Which change occurs faster between the anisotropic deformation and the OM deflection? The cantilever experiments for $\mathrm{As}_{2} \mathrm{~S}_{3}$ performed using focused light, the results being given by the two points at $\sim 10^{5} \mathrm{~W} / \mathrm{cm}^{3}$ in Fig. 4 , can afford such a comparison. The deformation and the deflection have been probed, in situ, through transmitted light patterns and the apex displacement, respectively. For instance, Fig. 5 shows transmitted light patterns (a) when an exposure of linearly polarized light is just started and (b) when an anisotropic deformation is already formed at a 20-min exposure. The transmitted light pattern is noisy, probably reflecting cantilever deflection and so forth. Nevertheless, the cantilever deflection has appeared faster than the anisotropic deformation, with a typical time-scale ratio of 1:10 for $\mathrm{S} 3$ in Table I. That is, the OM effect always occurs faster than the anisotropic deformation. Note that this tendency is consistent with the time scales compared in Fig. 7, which is obtained for the different substrates of glass and mica.

Figure 6 compares spectral dependences of the deformation efficiency and the $\mathrm{OM}$ displacement $\Delta$ in $\mathrm{As}_{2} \mathrm{~S}_{3}$ and Se. Here, the efficiency of anisotropic deformations is defined as that in the previous study, ${ }^{25}$ i.e., an inverse of absorbed photon numbers for producing a fixed $\mathrm{M}$-shape deformation, in which the central valley has the same level with an original flat surface. For instance, the maximal efficiency of $10^{-14}$

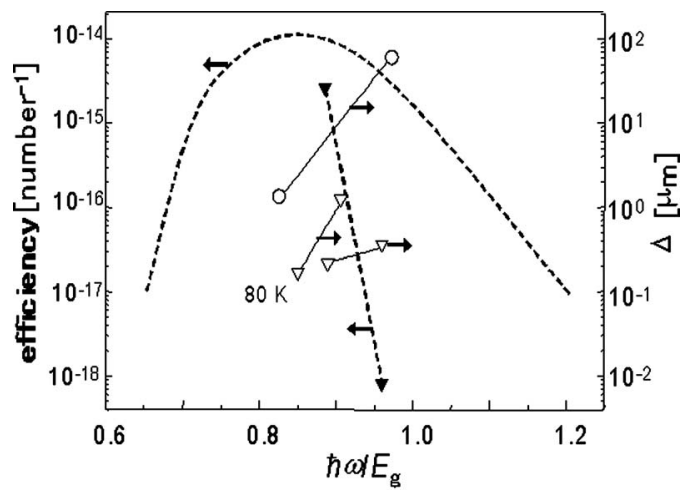

FIG. 6. Spectral dependences of a deformation efficiency (dashed lines with the left-hand side axis) in $0.7-\mu \mathrm{m}$ thick $\mathrm{Se}(\boldsymbol{\nabla})$ and the optomechanical displacement $\Delta$ (solid lines with the right-hand side axis) in 5 - $\mu \mathrm{m}$ thick $\mathrm{As}_{2} \mathrm{~S}_{3}\left({ }^{\circ}\right)$ and $5-\mu \mathrm{m}$ thick $\mathrm{Se}(\nabla)$ as a function of a normalized energy $\hbar \omega / E_{g}$. The deformation efficiency in $\mathrm{As}_{2} \mathrm{~S}_{3}(2-\mu \mathrm{m}$ thick) is replotted from Ref. 25 as a single dashed line for comparison. All the results, except for Se at $80 \mathrm{~K}$, are obtained at room temperature. 


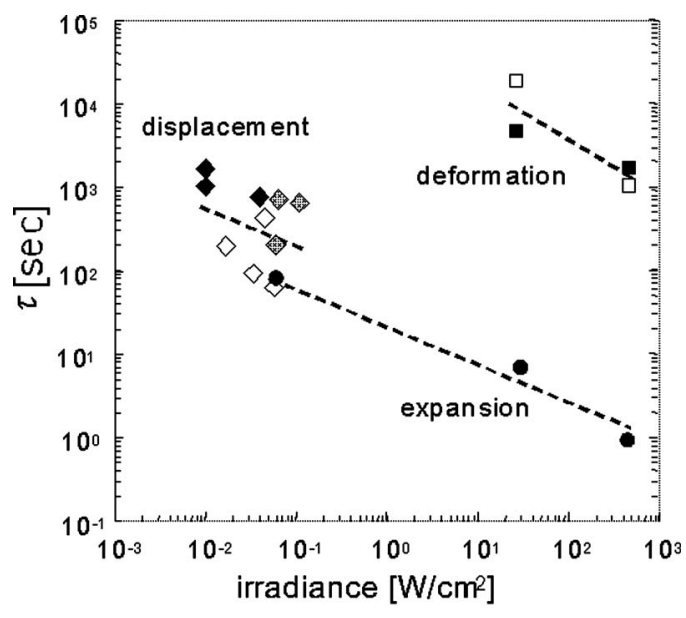

FIG. 7. Growing time scales of isotropic photoexpansion (circles), anisotropic deformation (squares), and optomechanical displacement (diamonds) in $\mathrm{As}_{2} \mathrm{~S}_{3}$ films with thicknesses of $\sim 5 \mu \mathrm{m}$ (solid symbols), $\sim 1 \mu \mathrm{m}$ (open symbols), and $\sim 0.3 \mu \mathrm{m}$ (gray symbols) upon exposures to $2.3 \mathrm{eV}$ light. The straight lines are obtained through the least-square fitting procedure.

means that the deformation needs absorption of $10^{14}$ photons per a typical light spot of $\sim 10 \mu \mathrm{m}$ in diameter, which reads very roughly $\sim 10^{23}$ photons $/ \mathrm{cm}^{3}$. For $\Delta$, the absolute value is plotted. On the other hand, the horizontal axis is the photon energy $\hbar \omega$ normalized to the Tauc gap $E_{g}{ }^{2,8}$ which becomes greater at lower temperatures. Note again that $\Delta$ is irrespective of the light intensity.

We see, for these materials at around $\hbar \omega / E_{g} \approx 0.9$, that the deformation efficiency (dashed lines) and the OM displacement (solid lines) show opposite variations. With an increase in $\hbar \omega / E_{g}$, the deformation efficiency becomes smaller and the displacement greater, the latter being consistent with the previous results. ${ }^{18-20}$ Here, the deformation reduction at higher energies may reflect a penetration-depth effect. A similar spectral dependence has been observed for the isotropic expansion. ${ }^{25}$ By contrast, the displacement enhancement at higher energies can be ascribed to a pure photon-energy effect.

Figure 7 compares growing time scales $\tau$ as a function of irradiance $I$ for the isotropic photoexpansion [Fig. 1(a)], the anisotropic deformation [Fig. 1(b)], and the OM displacement in $\mathrm{As}_{2} \mathrm{~S}_{3}$ upon exposures to $2.3 \mathrm{eV}$. The photoexpansion is assumed to grow exponentially, as suggested previously, ${ }^{25}$ and its time constant is plotted. For the anisotropic deformation, the time scale is taken to be the exposure time, when the anisotropic deformation changes to featureless patterns. ${ }^{25}$ For the OM effect, $\tau$ is determined through fitting the stretched exponential curve as described in Sec. III B. The data, while these being obtained through the leastsquare fitting procedure, are more-or-less scattered, probably reflecting transitory behaviors and so forth. Because of these different definitions of $\tau$, we can compare only rough features.

There are, however, notable features common to and different among the three time scales. The common tendency is $\tau \propto I^{-0.5}$, which implies that photoelectronic excitation processes in all the phenomena are governed by bimolecular recombination. ${ }^{8}$ On the other hand, the anisotropic deforma- tion gives appreciably (2-3 orders) longer time scales than that of the OM effect, which is similar to that of the isotropic deformation. In the previous work, ${ }^{25}$ the present authors connect the time scales of the isotropic expansion and the anisotropic deformation to related material-flow distances, which are the film thickness and the laser spot diameter, respectively. Nevertheless, underlying atomic forces and fluidity are assumed to be the same kinds for these deformations.

\section{DISCUSSION}

The present results strongly suggest different origins of the OM effect and the anisotropic deformation. As shown in Figs. 2 and 4, the light-intensity dependences are clearly different. The spectral dependences, shown in Fig. 6, are opposite at around the subgap region. Figure 7 shows that the growing time scales appear to be different by $2-3$ orders. And, the sharpest difference is that the film extension in the $\mathrm{OM}$ effect and the material flow in the anisotropic deformation occur in different directions in respect of the light polarization. That is, the deflection and the deformation are greater in perpendicular and parallel directions to the electric field. What are the microscopic origins?

For photoinduced optical anisotropies such as dichroism and birefringence, Fritzshce has proposed a guiding model. ${ }^{34}$ A chalcogenide glass is assumed to be composed of microscopic anisotropic elements having random orientations, which makes the glass macroscopically isotropic. When the glass is exposed to linearly polarized light, the light efficiently excites anisotropic elements having optical axes being nearly parallel to the electric field, and the elements may relax with thermal activations to other orientations. Accordingly, after the exposure, the glass possesses anisotropic properties. This model is successful in understanding the socalled negative anisotropy, ${ }^{9,10,34}$ e.g., negative dichroism $\alpha(\|)<\alpha(\perp)$, where $\alpha(\|)$ and $\alpha(\perp)$ are absorption coefficients parallel and perpendicular to the electric field of excitation light. Nevertheless, several structural models have been proposed for the anisotropic element, ${ }^{9}$ which remains to be studied.

In the previous work, ${ }^{25}$ the authors have adopted a segmental-layer model for interpreting the anisotropic $\mathrm{M}$-shaped deformation in $\mathrm{As}_{2} \mathrm{~S}_{3}$. The anisotropic element is assumed to be aligned low-dimensional segments with an extent of $\sim 2 \mathrm{~nm}$, e.g., stacked layers in $\mathrm{As}_{2} \mathrm{~S}_{3}$, and the orientations are changed by linearly polarized excitations and successive thermal relaxation. The process may resemble thermal nucleation and growth of crystallites. Note that this model is consistent with other observations such as the photoinduced oriented crystallization in Se, for which folded chains seem to be responsible. ${ }^{9,10,12}$ We assume that, upon prolonged illumination, intrasegmental disordering occurs, which causes with combination of photoinduced fluidity ${ }^{30-32}$ the anisotropic M-shaped deformation. ${ }^{25}$ On the other hand, the isotropic expansion is assumed to reflect structural disordering. ${ }^{28}$ These models are applicable also to explain the observation for Se in Fig. 1, as illustrated previously 
(Fig. 8 in Ref. 25). However, quantitative aspects, such as the similar magnitudes of the deformations in $\mathrm{As}_{2} \mathrm{~S}_{3}$ and $\mathrm{Se}$ (Fig. 2), remain to be puzzling.

This segmental model may explain also the OM effect. Under illumination of linearly polarized light, the model postulates that distorted layers in $\mathrm{As}_{2} \mathrm{~S}_{3}$ and entangled chains in Se tend to straighten in the directions perpendicularly to the electric field (Fig. 8 in Ref. 25). In the OM effect, if the field is perpendicular to the cantilever axis, the straightening will lengthen the chalcogenide film, giving rise to a greater cantilever deflection, which is consistent with the observation (Fig. 3). However, this model appears to be inconsistent with the opposite spectral dependences of the deformation efficiency and the OM effect (Fig. 6). Otherwise, we may assume that the deformation spectrum is governed by the photoinduced fluidity, ${ }^{30-32}$ while its spectral dependence has not been known.

For interpreting the OM effect, Krecmer et al. assume a photoinduced rotation of chalcogen atoms. ${ }^{15}$ The model has been proposed previously for interpreting photoinduced optical anisotropy ${ }^{35}$ If $\mathrm{S}$ atoms in specified $=\mathrm{As}-\mathrm{S}-\mathrm{As}=$ linkages are selectively excited by linearly polarized light, a resulting rotational motion of the $\mathrm{S}$ atom may provide macroscopic uniaxial lengthening and shortening of the chalcogenide film in response to the polarization directions. The change may deflect the cantilever, as observed. Nevertheless, in pure Se films, which also exhibit the OM effect [Fig. 3(b)], there exist no fulcra such as As in =As-S-As= linkages. All Se atoms are movable in similar degrees, and accordingly, applications of the chalcogen-rotation model seem to be difficult.

Does the OM effect arise only from some polarizationselective atomic motions? There may be other possibilities, which arise from polarization-dependent light scattering known in optics. ${ }^{36}$ On material surfaces, there exist small dusts and scratches, which scatter light preferentially in the directions perpendicular to the electric field, the process being known as the Rayleigh-Gans scattering. Scattered light propagates near the surface as leaky waves, and in highlyrefractive films, the light may be converted to guided waves. ${ }^{37}$ These scattered waves can interfere with incident light, giving rise to interference fringes, which align parallel to the electric-field direction. And, in chalcogenide glasses, the fringe will produce refractive-index fringes through photoinduced refractive-index changes. ${ }^{1-3,6}$ The index fringe can then operate as grating couplers, ${ }^{37}$ which further enhance scattered-light intensity. Under such circumstances, the scattering and coupler theories predict that the scattered light intensities propagating in perpendicular and parallel to the electric field are, very roughly, 10 and 1 under an incident light intensity of 100. Actually, such fringes have been detected as photoinduced $\mathrm{Ag}$ modulations in amorphous $\mathrm{Ag}$ As-S films. ${ }^{38}$

The scattered light may cause seemingly polarizationdependent effects. For instance, in $\mathrm{As}_{2} \mathrm{~S}_{3}$ and $\mathrm{Se}$, such fringes will convert transitory volume expansion ${ }^{39}$ to anisotropic. The expansion may be proportional to the number of interference fringes, which is greater when light polarization is perpendicular to a cantilever axis, and accordingly, the perpendicular polarization can provide a greater cantilever deflection. Otherwise, more generally, the guided light may provide greater thermal expansion, electrostriction, ${ }^{40}$ and radiation pressure. $^{41}$ However, these intrinsically scalar changes cannot explain the contracting bending in Se under exposures to the parallel polarization [Fig. 3(b)]. This polarization-dependent scattering model also faces to a difficulty in explaining the cantilever deflection induced by changing polarization directions of focused light spots (Fig. 4).

In short, these considerations show that atomic mechanisms of the OM effect remain to be speculative. In addition, many problems remain unresolved. For instance, why does not the transitory deflection change with the light intensity (Fig. 4)? What is the meaning of the stretched exponential transients having $\beta_{2} \approx 0.6$ (Ref. 42)?

\section{v. CONCLUSIONS}

Comparisons of the optomechanical effect and the photoinduced anisotropic deformation in two kinds of chalcogenide glasses, $\mathrm{As}_{2} \mathrm{~S}_{3}$ and $\mathrm{Se}$, manifest the following two features: One is that both the phenomena appear in these materials, which suggests that the phenomena are inherent to the covalent chalcogenide glass. The other is that the two phenomena exhibit markedly different features such as the light-intensity dependences, which suggest different photostructural processes. The optomechanical effect may arise from short-range atomic motions, the detail being speculative at present. On the other hand, the anisotropic deformation is assumed to be a manifestation of photoinduced atomic alignments and fluidity, as previously proposed. ${ }^{25}$ Similar photoinduced mechanical anisotropies appear also in oxide glasses and organic polymers, ${ }^{13,14,43}$ for which comparative and/or unified studies will be scientifically and technologically valuable.

\section{ACKNOWLEDGMENT}

The authors would like to thank S. R. Elliott and P. Krecmer for private correspondences.

\footnotetext{
${ }^{1}$ K. Shimakawa, A. Kolobov, and S. R. Elliott, Adv. Phys. 44, 475 (1995). ${ }^{2}$ M. A. Popescu, Non-Crystalline Chalcogenides (Kluwer Academic, Dordrecht, 2000).

${ }^{3}$ Photo-induced metastability in Amorphous Semiconductors, edited by A. V. Kolobov (Wiley-VCH, Weinheim, 2003).

${ }^{4}$ J. P. De Neufville, S. C. Moss, and S. R. Ovshinsky, J. Non-Cryst. Solids 13, 191 (1974).

${ }^{5}$ H. Hamanaka, K. Tanaka, A. Matsuda, and S. Iizima, Solid State Commun. 19, 499 (1976).

${ }^{6}$ K. Tanaka and Y. Ohtsuka, J. Appl. Phys. 49, 6132 (1978).

${ }^{7}$ K. Tanaka, N. Kawakami, and A. Odajima, Jpn. J. Appl. Phys., Part 120 , 1874 (1981).

${ }^{8}$ N. F. Mott and E. A. Davis, Electronic Processes in Non-Crystalline Materials, 2nd ed. (Clarendon, Oxford, 1979), Chaps. 6, 9, and 10.

${ }^{9} \mathrm{~K}$. Tanaka, in Handbook of Advanced Electronic and Photonic Materials and Devices, edited by H. S. Nalwa (Academic, San Diego, 2001), Vol. 5, Chap. 4.

${ }^{10} \mathrm{~V}$. M. Lyubin and M. L. Klebanov, in Photo-Induced Metastability in Amorphous Semiconductors, edited by A. V. Kolobov (Wiley-VCH, Weinheim, 2003), Chap. 6.

${ }^{11}$ V. G. Zhdanov and V. K. Malinovskii, Sov. Tech. Phys. Lett. 3, 387 (1977).

${ }^{12}$ K. Ishida and K. Tanaka, Phys. Rev. B 56, 2006 (1997).
} 
${ }^{13}$ H. Jain and J. Optoelectron, Adv. Mater. 5, 5 (2003).

${ }^{14}$ K. Tanaka, J. Non-Cryst. Solids 352, 2580 (2006).

${ }^{15}$ P. Krecmer, A. M. Moulin, R. J. Stephenson, T. Rayment, M. E. Welland, and S. R. Elliott, Science 277, 1799 (1997).

${ }^{16}$ A. Saliminia, T. V. Galstian, and A. Villeneuve, Phys. Rev. Lett. 85, 4112 (2000).

${ }^{17}$ M. Stuchlik, P. Krecmer, and S. R. Elliott, J. Optoelectron. Adv. Mater. 3, 361 (2001).

${ }^{18}$ M. Stuchlik, P. Krecmer, and S. R. Elliott, in Photo-Induced Metastability in Amorphous Semiconductors, edited by A. V. Kolobov (Wiley-VCH, Weinheim, 2003), Chap. 7.

${ }^{19}$ M. Stuchlik, P. Krecmer, and S. R. Elliott, IEE Proc.: Sci., Meas. Technol. 151, 131 (2004).

${ }^{20}$ M. Stuchlik and S. R. Elliott, J. Non-Cryst. Solids 353, 250 (2007).

${ }^{21}$ M. L. Trunov and V. S. Bilanich, J. Optoelectron. Adv. Mater. 6, 157 (2004).

${ }^{22}$ K. E. Asatryan, B. Paquet, T. Galstian, and R. Vallée, Phys. Rev. B 67, 014208 (2003).

${ }^{23}$ K. E. Asatryan, T. Galstian, and R. Vallée, Phys. Rev. Lett. 94, 087401 (2005).

${ }^{24}$ K. Tanaka and A. Odajima, Solid State Commun. 43, 961 (1982).

${ }^{25}$ K. Tanaka and H. Asao, Jpn. J. Appl. Phys., Part 1 45, 1668 (2006).

${ }^{26}$ M. Fernandez-Guasti, E. Haro-Poniatowski, and S. Camacho-Lopez, Appl.
Opt. 31, 3453 (1992).

${ }^{27}$ H. Hisakuni and K. Tanaka, Appl. Phys. Lett. 65, 2925 (1994).

${ }^{28}$ K. Tanaka, A. Saitoh, and N. Terakado, J. Optoelectron. Adv. Mater. 8, 2058 (2006).

${ }^{29}$ V. Palyok, I. A. Szabo, D. L. Beke, and A. Kikineshi, Appl. Phys. A: Mater. Sci. Process. 74, 683 (2002).

${ }^{30}$ H. Hisakuni and K. Tanaka, Science 270, 974 (1995).

${ }^{31}$ K. Tanaka, in Photo-Induced Metastability in Amorphous Semiconductors, edited by A. V. Kolobov (Wiley-VCH, Weinheim, 2003), Chap. 5.

${ }^{32}$ K. Tanaka, C. R. Chim. 5, 805 (2002).

${ }^{33}$ H. Koseki and A. Odajima, Jpn. J. Appl. Phys., Part 1 21, 424 (1982).

${ }^{34}$ H. Fritzsche, Phys. Rev. B 52, 15854 (1995).

${ }^{35}$ I. Janossy, J. Hajto, and W. K. Choi, J. Non-Cryst. Solids 90, 529 (1987).

${ }^{36}$ H. C. van de Hulst, Light Scattering by Small Particles (Dover, New York, 1981), Chap. 6.

${ }^{37}$ Integrated Optics, 2nd ed., edited by T. Tamir (Springer, Berlin, 1979), Chaps. 2 and 3.

${ }^{38}$ T. Gotoh and K. Tanaka, J. Appl. Phys. 89, 4703 (2001).

${ }^{39}$ Y. Ikeda and K. Shimakawa, J. Non-Cryst. Solids 338-340, 539 (2004).

${ }^{40}$ P. G. Datskos, S. Rajic, and I. Datskou, Appl. Phys. Lett. 73, 2319 (1998).

${ }^{41}$ A. Casner and J.-P. Delville, Phys. Rev. Lett. 87, 054503 (2001).

${ }^{42}$ J. C. Phillips, J. Non-Cryst. Solids 352, 4490 (2006).

${ }^{43}$ Y. Yu, M. Nakano, and T. Ikeda, Nature (London) 425, 145 (2003). 